\title{
GENERALIZATIONS OF A THEOREM OF SIERPINSKI AND ZYGMUND ON CONTINUOUS FUNCTIONS ${ }^{1}$
}

\author{
C. J. PIPES
}

1. Introduction. W. Sierpinski and A. Zygmund have shown $[1],{ }^{2}$ with the use of the continuum hypothesis, that there is a real function that is not continuous on any nondenumerable set relative to the set. In direct contrast with this result $\mathrm{H}$. Blumberg has shown [2] that every real function is continuous on a dense, denumerable set relative to the set.

Suppose denumerable ${ }^{3}$ sets are considered as negligible in the definition of continuity; i.e., $f(x)$ is $d$-continuous at $x$ if for every $\epsilon>0$ there is an open interval $I$ containing $x$ and a denumerable set $D$ such that for every $y \in I-D,|f(x)-f(y)|<\epsilon$. If continuity is replaced by $d$-continuity it becomes clear that one of the above two theorems is true and the other false. It is shown here that the analogue of the Sierpinski theorem is valid; i.e., there is a real function that is not $d$-continuous on any nondenumerable set relative to the set. Indeed, this is a special case of a general result relating to a class of types of negligible sets.

2. $\pi$-neglect. Consider an abstract set property $\pi$ defined by the following postulates.

DEFINITION 1. $\pi$ is a set property such that:

(1) Every subset, including the empty set, of a set that has the property likewise has the property.

(2) The union of a denumerable number of sets that have the property likewise has the property.

(3) Every set consisting of a single point has the property.

(4) The continuum does not have the property.

Any set property which satisfies these postulates will be called a $\pi$-property and the individual sets of the corresponding class of sets defined by this property shall be called $\pi$-sets. Sets which do not have the property $\pi$ will be designated $\bar{\pi}$-sets. Then, for any $\pi$-property, the continuum is a $\bar{\pi}$-set.

Presented to the Society, November 24, 1951; received by the editors March 22' 1951.

1 This paper is taken from a part of the author's dissertation written in partial fulfillment of the $\mathrm{Ph}$.D. requirement at the University of Oklahoma.

2 Numbers in brackets refer to the list of references.

${ }^{3}$ The term denumerable is used in the broad sense to include the empty set and finite sets as well as denumerably infinite sets. 
Clearly, the totality of denumerable sets forms a class of $\pi$-sets where the $\pi$-property is denumerability. Two other common classes of sets that are classes of $\pi$-sets are the class of sets of the first category and the class of sets of measure zero.

In order to localize the character of any given set it is convenient to make the following definition.

Definition 2. For any $\pi$-property and set $S$ a point $x$ is said to be a $\bar{\pi}$-point of $S$ if for every open interval $I$ containing $x, I \cap S$ is a $\bar{\pi}$-set. The set of $\bar{\pi}$-points of $S$ will be denoted by $S_{\bar{\pi}}$.

If $x$ is not a $\bar{\pi}$-point of $S$, it is called a $\pi$-point of $S$; i.e., $x$ is a $\pi$-point of $S$ if there exists an open interval $I$ containing $x$ such that $I \cap S$ is a $\pi$-set. The set of $\pi$-points of $S$ will be denoted by $S_{x}$.

As a consequence of this definition, the following result is easily obtained.

Lемма 1. For every set property $\pi$ and set $S$ the set $S \cap S_{\pi}$ is a $\pi$-set.

Proof. Let $x \in S \cap S_{\pi}$. There is an open interval $I$, with rational end points, containing $x$ such that $I \cap S$ is a $\pi$-set. Since every point of $S \cap S_{\pi}$ is in such an interval and since these intervals are denumerable in number, $S \cap S_{\pi}$ is contained in the union of a denumerable number of $\pi$-sets. Hence, $S \cap S_{\pi}$ is a $\pi$-set.

If, for any $\pi$-property, $\pi$-sets are considered as negligible sets the following generalization of continuity is obtained.

Definition 3. For every set property $\pi, f(x)$ is $\pi$-continuous at a point $y$ if for every $\epsilon>0$ there is an open interval $I$ containing $y$ such that the set of points $x \in I$ for which $|f(x)-f(y)| \geqq \epsilon$ is a $\pi$-set.

It will be shown that there is a real function that is not $\pi$-continuous on any $\bar{\pi}$-set relative to the set. Theorem 1 is the principal tool in the proof of this result. Essential to these results is the following definition.

Definition 4. For every set property $\pi$, a set $S$ is $\bar{\pi}$-dense-in-itself if and only if every point in $S$ is a $\bar{\pi}$-point of $S$.

THEOREM 1. For every set property $\pi$, if a set $S$ is $\bar{\pi}$-dense-in-itself then $f(x) \pi$-continuous on $S$ relative to $S$ implies that $f(x)$ is continuous on $S$ relative to $S$.

Proof. Let $S$ be $\bar{\pi}$-dense-in-itself and let $f(x)$ be $\pi$-continuous on $S$ relative to $S$. Suppose $f(x)$ is not continuous on $S$ relative to $S$. Then there is a $k>0$ and an $x \in S$ such that every open interval $I$ containing $x$ contains a $y \in S$ such that $|f(x)-f(y)| \geqq 2 k$. Let $I_{x}$ and $y$ be any such interval and point. Now $f(x)$ is $\pi$-continuous at $y$ relative to $S$; hence, there is an open interval $I_{y}$ containing $y$ and a $\pi$-set 
$E$ such that for every $z \in I_{y} \cap(S-E),|f(y)-f(z)|<k$. Since any subinterval of $I_{y}$ that contains $y$ satisfies the last condition, $I_{y}$ may be chosen so that $I_{y} \subset I_{x}$. Then for every $z \in I_{y} \cap(S-E)$,

$$
|f(x)-f(z)| \geqq|f(x)-f(y)|-|f(y)-f(z)|>2 k-k=k .
$$

Now every point of $S$ is a $\bar{\pi}$-point of $S$, so that $I_{y} \cap S$ is a $\bar{\pi}$-set and hence $I_{y} \cap(S-E)$ is a $\bar{\pi}$-set. Thus, in every open interval $I_{x}$ contalning $x$ there is a $\bar{\pi}$-subset $I_{y} \cap(S-E)$ of $S$ such that for every $z \in I_{y}$ $\cap(S-E),|f(x)-f(z)|>k$. This contradicts the hypothesis that $f(x)$ is $\pi$-continuous at $x$ relative to $S$. Therefore $f(x)$ is continuous at $x$ relative to $S$.

In addition to the preceding result, the following lemmas will be found useful.

Lemma 2. For every set property $\pi$, if $f(x)$ is $\pi$-continuous on a set $S$ relative to $S$, then $f(x)$ is $\pi$-continuous on any subset $T$ of $S$ relative to $T$.

Proof. Let $\epsilon>0$ and let $x \in T \subset S$. Then there is an open interval $I$ containing $x$ and a $\pi$-set $E$ such that for every $y \in I \cap(S-E)$

$$
|f(x)-f(y)|<\epsilon .
$$

Since $T \subset S$, for every $y \in I \cap(T-E)$

$$
|f(x)-f(y)|<\epsilon .
$$

LEMмA 3. For every set property $\pi$, if $f(x)$ is $\pi$-continuous on a $\bar{\pi}$-set $S$ relative to $S$ then there is a $\bar{\pi}$-subset $T$ of $S$ such that $f(x)$ is continuous on $T$ relative to $T$.

Proof. By Lemma 1 the points of $S$ not $\bar{\pi}$-points of $S$ form a $\pi$-set; hence, if $T$ is the set of points of $S$ that are $\bar{\pi}$-points of $S$, then $T$ is a $\bar{\pi}$-set. $T$ is also $\bar{\pi}$-dense-in-itself, for if $x \in T$ then every open interval $I$ containing $x$ is such that $I \cap S$, and hence $I \cap T$, is of type $\bar{\pi}$; therefore, $x$ is a $\bar{\pi}$-point of $T$. Then, by Lemma 2 and Theorem $1, f(x)$ is continuous on $T$ relative to $T$.

From the foregoing proof one easily sees the following:

CoROllaRY. For every set property $\pi$, if $f(x)$ is $\pi$-continuous on any set $S$ relative to $S$, then $f(x)$ is continuous on a set $T \subset S$ relative to $T$, where $S-T$ is a $\pi$-set.

With the aid of these results and the continuum hypothesis one may now prove the following:

THEOREM 2. For every set property $\pi$, there is a real function $h(x)$ that is not $\pi$-continuous on any $\bar{\pi}$-set relative to the set. 
Proof. Let $S$ be any $\bar{\pi}$-set and let $f(x)$ be any real function that is $\pi$-continuous on $S$ relative to $S$. By Lemma 3 there is a $\bar{\pi}$-subset $T$ of $S$ such that $f(x)$ is continuous on $T$ relative to $T$. From Definition 1 it is seen that for every $\pi$-property, denumerable sets are $\pi$-sets, hence, $T$ is nondenumerable. But, a function continuous on a nondenumerable set relative to the set can be extended to a Baire function on the set of all reals $[3$, p. 81]. Thus, there is a Baire function $g(x)$ on the reals that agrees with $f(x)$ on the nondenumerable set $T$. Hence, any function that is $\pi$-continuous on a $\bar{\pi}$-set relative to the set must agree with a Baire function on some nondenumerable set. The proof is then completed by finding a function $h(x)$ which does not agree with any Baire function on any nondenumerable set. This function can be found by a technique borrowed from Sierpinski [4, p. 116], which is given for completeness.

The set of Baire functions has cardinal number $C$, the cardinal number of the continuum. Let $\Omega$ be the smallest nondenumerable ordinal number. By the continuum hypothesis and the well-ordering theorem, the set of Baire functions and the set of real numbers may be put in one-to-one correspondence with the ordinal numbers $\alpha<\Omega$. For each $\alpha<\Omega$ let $g_{\alpha}(x)$ and $x_{\alpha}$ be the Baire function and real number mated with $\alpha$. Moreover, let $E_{\alpha}$ be the set of all real numbers $g_{\beta}\left(x_{\alpha}\right)$ for which $\beta<\alpha$. Then $E_{\alpha}$ is denumerable. Let $x_{\xi \alpha}$ be the first element of the transfinite sequence $x_{\gamma}, \gamma<\Omega$, which does not belong to $E_{\alpha}$. For every $\alpha<\Omega$, let $h\left(x_{\alpha}\right)=x_{\xi \alpha}$. Then $h(x)$ is defined everywhere and, for every $\alpha<\Omega, h\left(x_{\alpha}\right) \notin E_{\alpha}$. Now, let $\gamma<\Omega$. The definition of $h(x)$ implies that $h\left(x_{\alpha}\right) \neq g_{\gamma}\left(x_{\alpha}\right)$ for all $\alpha>\gamma$ so that $h(x)=g_{\gamma}(x)$ only on a denumerable set. Hence $h(x)$ does not agree with any Baire function on any nondenumerable set and the theorem is proved.

Lemma 2 allows Theorem 2 to be put in another form.

THEOREM 3. For every set property $\pi$ there is a real function $h(x)$ whose points of $\pi$-continuity on any set $S$ relative to $S$ form $a \pi$-set.

Proof. Let $h(x)$ be the function of Theorem 2. Suppose that the theorem is not true. Then there is a set $T$ such that the set $S \subset T$ of points of $\pi$-continuity of $h(x)$ on $T$ relative to $T$ forms a $\bar{\pi}$-set. Now by Lemma $2, h(x)$ is $\pi$-continuous on $S$ relative to $S$. Since $S$ is a $\bar{\pi}$-set, this contradicts Theorem 2 .

3. Approximate continuity. A significant generalization of continuity which uses the concept of negligible sets but which is not included in the $\pi$-classification above has been introduced by Denjoy [5, p. 165]. In defining "approximate continuity" at a point Denjoy considered as negligible any set of metric density zero at the point. 
More precisely, he defined a measurable function $f(x)$ to be approximately continuous at a point $x$ if for every $\epsilon>0$ the set of points $y$ for which $|f(x)-f(y)|<\epsilon$ has metric density one at $x$.

In order to extend the Sierpinski theorem to approximate continuity it is necessary to extend the definition of approximate continuity to arbitrary real functions. Two alternatives suggest themselves. The first is obtained by merely replacing "metric density" by "exterior metric density" in the above definition. This definition, while useful in certain considerations [6], does not possess some of the fundamental properties essential to this paper; hence, the second and more restrictive definition, first used by E. Kamke [7], will be used.

Definition 5. A real function $f(x)$ is approximately continuous at the point $x$ if for every $\epsilon>0$ the set of points $y$ for which $|f(x)-f(y)|$ $\geqq \epsilon$ has exterior metric density zero at $x$.

Using this definition, the following result will be obtained: there is a real function that is not approximately continuous on any set of positive exterior measure relative to the set. An essential tool in the proof of this result is Theorem 4, whose proof depends upon the following lemma.

Lemma 4. If $S$ is any set and $T \subset S$, then the set $E$ of points of $S$ at which the exterior metric density of $T$ exists and is equal to one is a Borel set relative to $S$.

Proof. Let $T_{n}$ be the set of points contained in sequences of intervals with lengths converging to zero in each of which the relative exterior measure of $T$ is less than $1-1 / n$. Each $T_{n}$ is of type $G_{\delta}$ so that $\cup_{n=1}^{\infty} T_{n}$ is of type $G_{\delta \sigma}$ and the intersection of its complement with $S$ is of type $F_{\sigma \delta}$ relative to $S$. But this last set is precisely the set of points of $S$ at which the exterior metric density of $T$ exists and is equal to one.

THEOREM 4. If $S$ is any set of positive exterior measure and if $f(x)$ is approximately continuous on $S$ relative to $S$, then there is a subset $T$ of $S$ of positive exterior measure such that $f(x)$ is a Baire function on $T$ relative to $T$.

Proof. Let $T$ be the points of $S$ at which the exterior metric density of $S$ exists and is equal to one. Since $S-T$ is of measure zero, $T$ is of positive exterior measure, and $f(x)$ is approximately continuous on $T$ relative to $T$. It will be shown that $f(x)$ is a Baire function on $T$ relative to $T$. In order to prove this, it will be shown that the set of points $x \in T$ for which $f(x)>K$ is a Borel set relative to $T$ for every value of $K$. 
Let $K$ be any real number and let $E$ be the set of points $x \in T$ for which $f(x)>K$. Let $A_{n} \subset E$ be the points of $E$ at which $f(x)>K+1 / n$. Then every point of $A_{n}$ is a point of exterior metric density one of $A_{n}$. To show this, let $\xi \in A_{n}$. Then $f(\xi)>K+1 / n$ and hence $f(\xi)>K+1 / n$ $+\delta$ for some $\delta>0$. Since $f(x)$ is approximately continuous at $\xi$ relative to $T$, the set of points $x \in T$ for which $|f(x)-f(\xi)| \geqq \delta$ has exterior metric density zero at $\xi$. But, $T$ has exterior metric density one at $\xi$; hence, the set of points $x \in T$ for which $|f(x)-f(\xi)|<\delta$ has exterior metric density one at $\xi$. This set is a subset of $A_{n}$ since $|f(x)-f(\xi)|<\delta$ implies that $f(x)>f(\xi)-\delta>K+1 / n+\delta-\delta=K+1 / n$; hence, $A_{n}$ has exterior metric density one at $\xi$. Furthermore, the exterior metric density of $A_{n}$ is zero at every point $\zeta \in T$ for which $f(\zeta)<K+1 / n$. This follows from the fact that $f(\zeta)<K+1 / n$ implies that $f(\zeta)<K$ $+1 / n-\delta$ for some $\delta>0$, and from the fact that the set of points $x \in T$ for which $|f(x)-f(\zeta)| \geqq \delta$, which includes $A_{n}$, has exterior metric density zero at $\zeta$.

Let $B_{n}$ be the set of points $x \in T$ for which $f(x)=K+1 / n$ and at which the exterior metric density of $A_{n}$ is one. Then $E_{n}=A_{n} \cup B_{n}$ is the set of points of $T$ at which the exterior metric density of $A_{n}$ is one, and hence, by Lemma 4 , is a Borel set relative to $T$. Then $\cup_{n=1}^{\infty} E_{n}$ is also a Borel set relative to $T$. One now has only to show that $E=\bigcup_{n=1}^{\infty} E_{n}$ to complete the proof of the theorem.

Now, for every $n, x \in E_{n}$ implies that $f(x) \geqq K+1 / n$ and hence $x \in E$. It follows that $\bigcup_{n=1}^{\infty} E_{n} \subset E$. Likewise, $x \in E$ implies that $f(x)>K$ which implies that $f(x)>K+1 / n$ for some value of $n$ and hence $x \in E_{n}$. Then $E \subset \bigcup_{n=1}^{\infty} E_{n}$. Thus, $E=\bigcup_{n=1}^{\infty} E_{n}$.

Since the set $T$ used in the proof of the above theorem is all of $S$ except for a set of measure zero, the following corollary has been proved.

CoRollary. If $f(x)$ is approximately continuous on $S$ relative to $S$, there is a set $T \subset S$ such that $m(S-T)=0$ and such that $f(x)$ is a Baire function on $T$ relative to $T$.

With the aid of Theorem 4 and using the continuum hypothesis, one may now prove the following analogue of the Sierpinski theorem.

THEOREM 5. There is a function that is not approximately continuous on any set of positive exterior measure relative to the set.

Proof. Let $S$ be any set having positive exterior measure and let $f(x)$ be approximately continuous on $S$ relative to $S$. Then, by Theorem 4, there is a subset $T$ of $S$ of positive exterior measure such that $f(x)$ is a Baire function on $T$ relative to $T$. But $T$, being of posi- 
tive exterior measure, is nondenumerable and it is known [3, p. 81] that any Baire function on a nondenumerable set can be extended to a Baire function on the reals. Accordingly, there is a Baire function $g(x)$ on the reals that agrees with $f(x)$ on the nondenumerable set $T$. The proof may be completed by finding a function $h(x)$ that does not agree with any Baire function on any nondenumerable set. This was done in Theorem 2.

The function $h(x)$ found above is characterized somewhat better by Theorem 6 which depends upon the following lemma.

LEMMA 5. If $H$ is the set of points of $S$ at which $h(x)$ is approximately continuous relative to $S$, then $h(x)$ is approximately continuous on $H$ relative to $H$.

Proof. Since $h(x)$ is approximately continuous on $H$ relative to $S$, for every $\epsilon>0$ and $y \in H$, the set $T$ of points $x \in S$ for which $|h(x)-h(y)| \geqq \epsilon$ has exterior metric density zero at $y$. Since $H \subset S$ the set $E$ of points $x \in H$ for which $|h(x)-h(y)| \geqq \epsilon$ is a subset of $T$ and therefore has exterior metric density zero at $y$. Then $h(x)$ is approximately continuous on $H$ relative to $H$.

THEOREM 6. There is a function whose points of approximate continuity on any set $S$ relative to $S$ form a set of measure zero.

Proof. By Theorem 5 there is a function $h(x)$ that is not approximately continuous on any set of positive exterior measure relative to the set. Suppose however that there is a set $S$ such that the set $T$ of points of $S$ at which $h(x)$ is approximately continuous relative to $S$ is of positive exterior measure. Then, by Lemma $6, h(x)$ is approximately continuous on $T$ relative to $T$, contradicting Theorem 5 .

\section{References}

1. W. Sierpinski and A. Zygmund, Sur une fonction que est discontinue sur tout ensemble de puissance du continu, Fund. Math. vol. 10 (1927).

2. H. Blumberg, New properties of all real functions, Trans. Amer. Math. Soc. vol. 24 (1922).

3. W. Sierpinski, Sur l'extension des fonctions de Baire definies sur les ensembles linéaires quelconques, Fund. Math. vol. 16 (1930).

4. - Hypothèse du continu, Warszawa-Lwów, 1934.

5. A. Denjoy, Sur les fonctions dérivées sommables, Bull. Soc. Math. France vol. 43 (1915).

6. C. Goffman, Proof of a theorem of Saks and Sierpinski, Bull. Amer. Math. Soc. vol. 54 (1948).

7. E. Kamke, Zur Definition der approximativ stetigen Funktionen, Fund. Math. vol. 10 (1927).

\section{SOUTHERN METHODIST UNIVERSITY}

